\title{
Composition and Structure of Aquatic Macroinvertebrate Communities in Mining Areas Streams (Côte d'Ivoire, West Africa)
}

\author{
Ouattara Dongui Séniva ${ }^{1}$, Tapé Logboh David ${ }^{1} \&$ Edia Oi Edia ${ }^{1}$ \\ ${ }^{1}$ Laboratoire d'Environnement et de Biologie Aquatique, UFR-Sciences et Gestion de l'Environnment, Nangui \\ Abrogoua' University, Abidjan 02, Côte d'Ivoire
}

Correspondence: Ouattara Dongui Séniva, Laboratoire d'Environnement et de Biologie Aquatique, UFR-Sciences et Gestion de l'Environnement, Nangui Abrogoua' University, 02 BP 801, Abidjan 02, Côte d'Ivoire.

Received: April 12, 2020

doi:10.20849/jess.v3i1.836
Accepted: May 26, 2020

Online Published: December 15, 2020

URL: https://doi.org/10.20849/jess.v3i1.836

\begin{abstract}
The existence of mining areas for decades could potentially affect aquatic environments and human health. This study allowed assessing the effects of mining activities on streams in three mining areas (Hiré, Lauzoua and Tortiya). Macroinvertebrates were collected on eight campaigns at eight stations using a hand-net, an Ekman grab and surber net. Environmental variables were recorded also. In this study the conductivity values were higher in the locality of Hiré. It was between $154.2 \mu \mathrm{S} / \mathrm{cm}$ (Tchindégri station) and $1753 \mu \mathrm{S} / \mathrm{cm}$ (Tributary Gbloh station). The highest temperature values were recorded in the stations of Tortiya locality $\left(24^{\circ} \mathrm{C}\right.$ : Bou $2 ; 33.1{ }^{\circ} \mathrm{C}$ : Bou 1). A total of 184 taxa distributed among nine classes, 20 orders, 80 families and comprising 14401 individuals were recorded. Insecta were the most diversified class in macroinvertebrate community (84\% of taxa collected, eight orders and 59 families). Ecological indices (Shannon Weiner, rarefied richness, Pielou's evenness) were significantly low at Lauzoua (N'Téko station) and Hiré (Tributary Gbloh station) (MannWhitney test, $\mathrm{P}<0.05$ ). Ecological indices showed no significant variation between the stations of the locality of Tortiya. The PCA had grouped the stations into four clusters. Conductivity, ammonium, phosphate, nitrites, and nitrates were significantly higher (Mann- Whitney test, P < 0.05) in cluster I (Tributary Gbloh station) Compared to other clusters. Eight taxa (Limnius sp., Liberonautes chaperi, Gordius sp., Phyllogomphus sp., Orectogyrus sp., Bezzia sp., Adenophlebiodes, Parasedodes sp.) were specific to Tchindégri station (Hiré) and six taxa (Naucoris sp., Amphiops sp., Hydrobius sp., Pseudobagous longulus, Culicinae and Gomphus sp.) were associated to the Bou 1 and Bou 2 stations (Tortiya).
\end{abstract}

Keywords: aquatic macroinvertebrates, composition, structure, mining activities, streams, Côte d' Ivoire

\section{Introduction}

Mining become widespread around the world due to the excessive price of certain metals and minerals. Metal prices are very high these days (World bank, 2019). In Côte d'Ivoire, gold and manganese are the two main minerals mined on an industrial scale (Fair Links, 2013). For example, four gold mining companies were created (Society of mining of Ity, Newcrest, Yaouré Mining and Tongon) since 2011 (Sodemi, 2012) as well as two manganese mining companies on an industrial scale (NABC and Boundoukou Manganese) (Koffi et al., 2014).

Human activities around streams such as mining has the most negative environmental impact, although seen as a beneficial activity in terms of socio-economic fallout (Bridge, 2004; Yapi et al., 2014). Processing activities resulting from mining exploration and development degrade the natural environment by polluting soil and water with tailings (Paquet, 2012). Indeed, among the mining wastes on earth, sulphide mine tailings are the most important and likely to produce acid mine drainage (AMD) (Dold, 2014). The AMD is responsible for the acid metalification of water. Water contaminated with AMD can be toxic to aquatic organisms (Battaglia et al., 2005; Mc Tammany, 2007).

The integrity of aquatic ecosystems is now based on quantify existing biological communities, including aquatic macroinvertebrates, which are the most commonly used bioindicators (Clarke et al. 2002). Indeed, these organisms are present at different water strata (Sanogo et al., 2014). They are good bioindicators because of their sedentary lifestyle, long-life cycle, great diversity and variable tolerance to pollution and habitat degradation (Moretti and Callisto, 2005). 
In many parts of the country, authorized and non-authorized mining are located, especially in Hiré, Lauzoua and Tortiya localities. Wastes from these mining activities can cover the surface of stream sediments, destroying habitat and reducing niches and nursery areas of aquatic macroinvertebrates (Jennings et al., 2008). In Côte d'Ivoire several studies explored the impact of mining activities based on the chemical quality of water (Coulibaly et al., 2009; Keumean et al., 2013; Yapi et al., 2014). However, there is a lack of study in Côte d'Ivoire using a biological community, especially macroinvertebrates, in order to assess the impact of mining activities on the rivers near the mining sites. This study will fill informations gap that will constitute a baseline for further studies.

This study aimed to inventory aquatic macroinvertebrates, to determine their composition, their structure and impacts of mining activities on communities of macroinvertebrates collected in streams in mining areas in order to assess their ecological quality.

\section{Materials and Methods}

\subsection{Environment Variables}

At each sampling station and each campaign, nine physicochemical parameters were measured using a variety of instruments. Conductivity was determined using a multi-parameter HANNA HI98703. A turbidimeter HANNA (HI 98703) was used to measure the turbidity and Dissolved oxygen was measured with an oximeter type HANNA HI9146. The $\mathrm{pH}$ and temperature were determined with a $\mathrm{pH}$ meter type HANNA HI991001. Water was also collected with bottle of $1 \mathrm{~L}$ at each sampling station for nutrients (ammonium, nitrate, nitrite, and phosphate) analysis in the laboratory.

\subsection{Study Areas and Sampling Stations}

The study was conducted in three mining areas in Côte d'Ivoire: Tortiya in the north (diamond mining), Hiré (gold mining) and Lauzoua (manganese mining) in the south of the country. Three streams were sampled in Hiré (Tributary Gbloh, Gbloh, Tchindégri) and lauzoua (Tributary Dougodou, Dougodou, N'Téko). In the town of Tortiya, the Bou stream was sampled. These localities were chosen according mining activities. Sampling stations (Figure 1) were selected according their accessibility, the permanence of water at any time and the potential presence of the impacts of mining activities. In each stream one station has been defined, except the Bou stream where two stations (Bou 1 and Bou 2) have been defined. The characteristics of the stations are listed in Table 1.

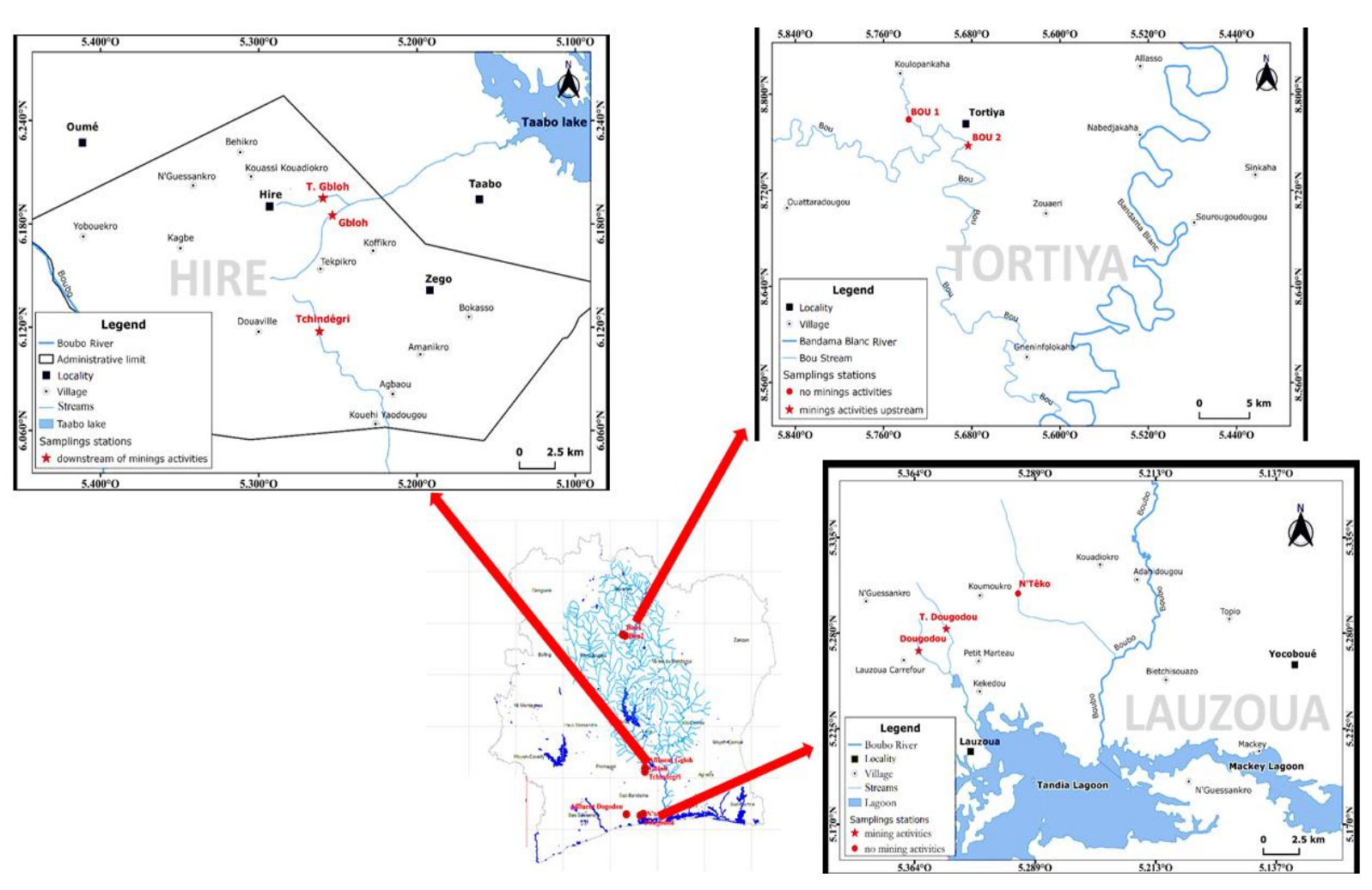

Figure 1. Maps of streams and sampling station 
Table 1. Characteristics of sampling stations

\begin{tabular}{|c|c|c|c|c|c|c|c|c|}
\hline LOCALITIES & \multicolumn{2}{|c|}{ TORTIYA } & \multicolumn{3}{|c|}{ HIRE } & \multicolumn{3}{|c|}{ LAUZOUA } \\
\hline Streams & & & Gbloh & Tributary Gbloh & Tchindegri & Dougodou & $\begin{array}{l}\text { Tributary } \\
\text { Dougodou }\end{array}$ & N'Teko \\
\hline Stations & Bou 1 & Bou 2 & Gbloh & Tributary Gbloh & Tchindegri & Dougodou & $\begin{array}{l}\text { Tributary } \\
\text { Dougodou }\end{array}$ & N'teko \\
\hline Latitude & 200619 & 387252 & 250551 & 249927 & 200615 & 238101 & 240225 & 245176 \\
\hline Longitude & 970058 & 595637 & 684486 & 685437 & 970060 & 582993 & 584411 & 546616 \\
\hline Altitude (m) & 300 & 343 & 191 & 168 & 189 & 4 & 1 & 4 \\
\hline $\begin{array}{c}\text { Land } \\
\text { occupation } \\
\text { and mining } \\
\text { activities }\end{array}$ & $\begin{array}{c}\text { Mahogany } \\
\text { plantation, food, } \\
\text { fishing, no } \\
\text { mining activity }\end{array}$ & $\begin{array}{l}\text { Fishing, washing } \\
\text { cars and beef, } \\
\text { abandoned } \\
\text { diamond mining } \\
\text { and artisanal } \\
\text { mining }\end{array}$ & $\begin{array}{c}\text { Teak, food } \\
\text { and cocoa } \\
\text { plantations, } \\
\text { artisanal } \\
\text { and } \\
\text { industrial } \\
\text { gold } \\
\text { mining }\end{array}$ & $\begin{array}{c}\text { Gold mining } \\
\text { company }\end{array}$ & $\begin{array}{c}\text { Cocoa } \\
\text { plantations and } \\
\text { abandoned } \\
\text { gold mining }\end{array}$ & $\begin{array}{c}\text { Cocoa } \\
\text { plantations, } \\
\text { village, } \\
\text { manganese } \\
\text { mining }\end{array}$ & $\begin{array}{c}\text { Manganese } \\
\text { mining, food and } \\
\text { cocoa } \\
\text { plantations, } \\
\text { village, }\end{array}$ & $\begin{array}{c}\text { Cocoa } \\
\text { plantations, } \\
\text { no mining } \\
\text { activity }\end{array}$ \\
\hline $\begin{array}{l}\text { Average } \\
\text { width (m) }\end{array}$ & 30 & 35 & 3 & 2,5 & 5 & 6 & 4 & 8 \\
\hline $\begin{array}{c}\text { Deep width } \\
\text { (m) }\end{array}$ & 3,5 & 4 & 0,9 & 1,5 & 1,1 & 4,5 & 4 & 5 \\
\hline Canopy (\%) & 0 & 0 & 90 & 5 & 20 & 50 & 5 & 80 \\
\hline
\end{tabular}

\subsection{Macroinvertebrate Sampling and Identification}

Macroinvertebrates were collected during eight campaigns (from November 2017 to January 2019) at each sampling station. Three gears were used to sample aquatic macroinvertebrates: Hand net $(250 \mu \mathrm{m} \mathrm{mesh}, 50 \mathrm{~cm}$ length), Surber net $(25 \times 20 \mathrm{~cm})$ and an Ekman grab $\left(0.115 \mathrm{~m}^{2}\right)$. The samples were sieved in the field through a $1 \mathrm{~mm}$ mesh sieve, and the material retained on the mesh was immediately fixed in $70 \%$ alcohol. In the laboratory, macroinvertebrates were identified to the lowest possible taxonomic level using a stereomicroscope Olympus SZ (40× magnification) and a series of identification keys (Monod, 1966; Déjoux et al., 1981; Day et al., 2001; Day et al., 2003; De Moor et al., 2003a; De Moor et al., 2003b; Stals \& De Moor, 2007 \& Tachet et al., 2010).

\subsection{Data Analysis}

Aquatic macroinvertebrates structure was described through taxonomic composition, rarefied richness, Shannon-Weiner index, Pielou's Evenness index, frequency of occurrence and Trichoptera, Plecoptera and Ephemeroptera index (EPT). Taxa richness was rarefied to eliminate any bias related to differences in abundances between samples (Heck et al., 1975; Edia et al., 2016). Calculations were performed using the lowest abundance (11 individuals for this study) found in all stations as the target number of individuals (Oksanen et al., 2013). Trichoptera, Plecoptera and Ephemeroptera index (EPT) was also determined at all stations with the aim of determining the impact of mining activities on the water quality of the studied stations. This index represents the relative abundance of these three groups among macroinvertebrates collected at all the stations.

The Frequency of occurrence (FO) was calculated at all sampling station. FO is the percentage of samples in which each taxon occurred. It was calculated according to Dajoz (2000) to gives some information on the number of taxa frequently met in each station without any indication on their quantitative importance (Lauzanne, 1976; Hyslop, 1980). 
In order to assess the structure of macroinvertebrate communities, in each mining area, between-stations variations of abovementionned indices were determined using Kruskal- Wallis and Mann-Whitney tests.

Principal Component Analysis (PCA) using the euclidean distance was performed to ordinates sampling stations according to environmental variables. A Hierarchical Classification Analysis (CAH) was performed on PCA axes in order to cluster sampling stations with similar environmental conditions. This analysis was carried out using the Factomine R and factoextra packages.

Variations of physico-chemical parameters and diversity indices (rarefied richness and Shannon-Weiner index) between clusters were assessed using Kruskal-Wallis and Mann- Whitney tests in order to characterize each group of stations. Before performing the comparison test, the normality of data was checked by Shapiro test.

Characteristic taxa of each group were determined through Indicator Value Method (Indval) (Dufrêne \& Legendre, 1997). This method matches information on species abundance and frequency of occurrence among groups. A Monte Carlo permutation test was employed to test significant associations of taxa and group of sites $(\mathrm{p}<0.05)$. The indicspecies package was used to perform this analysis. Data analyses were performed using R software version 3.6.3.

\section{Results}

\subsection{Environmental Variables}

Variations of temperature, conductivity, turbidity, $\mathrm{pH}$, dissolved oxygen, nitrate, nitrite, phosphate and ammonium between stations are summarized in Table 2. Temperature, $\mathrm{pH}$ and dissolved oxygen did not vary significantly between stations in the three localities (Kuskal-Wallis and Mann- Whitney tests, $p>0.05$ ). The highest and lowest values of temperature were observed in Tortiya locality respectively in Bou 2 station $\left(24{ }^{\circ} \mathrm{C}\right)$ and Bou 1 station (33.1). The $\mathrm{pH}$ varied from 6.15 (N'Téko station, Lauzoua) to 8.69 (Tributary Gbloh station, Hiré). Dissolveld oxygen values oscillated between $1.76 \mathrm{mg} / \mathrm{L}$ (Tributary Dougodou station, Lauzoua) and 12.71 $\mathrm{mg} / \mathrm{L}$ (Tributary Gbloh station, Hiré). Conductivity and turbidity did not vary significantly between the stations of Tortiya (Mann Whitney test $\mathrm{p}>0.05$ ). However, these parameters were statistically higher (Mann- Whitney test, $\mathrm{p}<0.05)$ respectively in Tributary Gbloh and Gbloh stations, in the locality of Hiré. Turbidity was significantly higher in Tributary Dougodou station, in Lauzoua locality. Concerning nutrients, excepted in Hiré locality, nitrate, nitrite, phosphate and ammonium did not varied significantly between stations in studied localities (Kruskal-Wallis and Mann- Whitney tests, $\mathrm{p}>0.05$ ).

Table 2. Environmental variables measured sampling stations in three mining areas (Côte d'Ivoire), $\mathrm{T}=$ temperature; $\mathrm{CND}=$ conductivity; $\mathrm{Turb}=$ turbidity $\mathrm{DO}=$ dissolved oxygen, $\mathrm{Phos}^{-}=$phosphate $\mathrm{Amo}=$ ammonium)

\begin{tabular}{|c|c|c|c|c|c|c|c|c|c|c|c|}
\hline Localities & Stations & Values & $\mathrm{T}\left({ }^{\circ} \mathrm{C}\right)$ & $\begin{array}{c}\text { CND } \\
(\mu \mathrm{S} / \mathrm{cm})\end{array}$ & $\mathrm{pH}$ & $\begin{array}{c}\mathrm{DO} \\
(\mathrm{mg} / \mathrm{L})\end{array}$ & $\begin{array}{c}\text { Turb } \\
\text { (NTU) }\end{array}$ & $\begin{array}{l}\text { Nitrate } \\
(\mathrm{mg} / \mathrm{L})\end{array}$ & $\begin{array}{l}\text { Nitrite } \\
(\mathrm{mg} / \mathrm{L})\end{array}$ & $\begin{array}{c}\mathrm{Amo} \\
(\mathrm{mg} / \mathrm{L})\end{array}$ & $\begin{array}{l}\text { Phos } \\
\text { (mg/L) }\end{array}$ \\
\hline \multirow{6}{*}{ Tortiya } & \multirow{3}{*}{ B 1} & Min & 25.3 & 108.1 & 6.31 & 2.62 & 16.5 & 2.2 & 0.009 & 0.08 & 0.04 \\
\hline & & Med & 30.5 & 150 & 6.82 & 4.84 & 56.73 & 4.25 & 0.017 & 0.415 & 0.215 \\
\hline & & Max & 33.1 & 199.3 & 7.22 & 8.58 & 170.67 & 22.4 & 0.055 & 6.15 & 3.64 \\
\hline & \multirow{3}{*}{ B 2} & Min & 24 & 65.9 & 6.52 & 2.4 & 4.3 & 3 & 0.015 & 0.1 & 0.03 \\
\hline & & Med & 28.2 & 107.3 & 6.68 & 4.71 & 22.21 & 5.05 & 0.02 & 1.185 & 0.303 \\
\hline & & Max & 30.3 & 412 & 7.45 & 12.5 & 308.67 & 41.6 & 0.154 & 5.65 & 1.93 \\
\hline \multirow{8}{*}{ Lauzoua } & \multirow{3}{*}{ D } & Min & 25.7 & 106.6 & 6.33 & 2.02 & 8.39 & 2.4 & 0.012 & 0.03 & 0.11 \\
\hline & & Med & 26.9 & 176.6 & 6.84 & 4.31 & 10.55 & 6.85 & 0.02 & 0.42 & 0.22 \\
\hline & & Max & 32.1 & 375 & 8.17 & 8.65 & 24.73 & 36.2 & 0.175 & 0.78 & 0.93 \\
\hline & \multirow{3}{*}{$\mathrm{TD}$} & Min & 25.3 & 101.5 & 6.23 & 1.76 & 19.4 & 1.2 & 0.014 & 0.038 & 0.03 \\
\hline & & Med & 27.2 & 192.1 & 6.93 & 4.2 & 39.35 & 3.5 & 0.031 & 0.54 & 0.295 \\
\hline & & Max & 28.4 & 452 & 7.64 & 8.8 & 225.67 & 31.4 & 0.072 & 0.79 & 2.61 \\
\hline & \multirow{2}{*}{ NT } & Min & 24.1 & 141.7 & 6.15 & 2.38 & 9.64 & 4.1 & 0.017 & 0.29 & 0.13 \\
\hline & & Med & 25.9 & 211.6 & 6.85 & 3.5 & 23.07 & 8.1 & 0.031 & 0.44 & 0.46 \\
\hline
\end{tabular}




\begin{tabular}{|c|c|c|c|c|c|c|c|c|c|c|c|}
\hline & & $\operatorname{Max}$ & 26.5 & 225.1 & 7.33 & 8.19 & 88.73 & 22.5 & 0.091 & 0.82 & 4.3 \\
\hline \multirow{9}{*}{ Hiré } & \multirow{3}{*}{ G } & Min & 25.4 & 316 & 6.65 & 2.37 & 28.43 & 2.4 & 0.008 & 0.05 & 0.12 \\
\hline & & Med & 26.4 & 579.8 & 6.92 & 5.71 & 101.28 & 3.8 & 0.025 & 0.745 & 0.74 \\
\hline & & Max & 28 & 946 & 7.69 & 9.74 & 710.33 & 8.2 & 0.087 & 0.97 & 2.01 \\
\hline & \multirow{3}{*}{ TG } & Min & 25 & 1162 & 6.54 & 6.71 & 102.3 & 34.1 & 0.205 & 2.05 & 0.97 \\
\hline & & Med & 27.5 & 1286 & 7.16 & 7.95 & 134.5 & 47.95 & 0.328 & 3.19 & 1.75 \\
\hline & & Max & 29.7 & 1753 & 8.69 & 8.47 & 189.3 & 59.6 & 0.512 & 4.88 & 2.77 \\
\hline & \multirow{3}{*}{$\mathrm{Tg}$} & Min & 24.4 & 154.2 & 6.36 & 6.57 & 42 & 27.4 & 0.1 & 0.11 & 0.2 \\
\hline & & Med & 26.15 & 194.2 & 7.13 & 8.26 & 73.89 & 40.35 & 0.117 & 0.251 & 1.24 \\
\hline & & Max & 29.9 & 241.3 & 7.24 & 12.71 & 120.33 & 50.3 & 0.19 & 0.59 & 1.84 \\
\hline
\end{tabular}

\subsection{Taxonomic Richness and Composition}

Table 3 shows macroinvertebrate composition of studied streams in three mining areas. A total of 176 different taxa (genus and species) belonging to nine classes (Insecta, Gastropoda, Malacostraceae, Acheata, Oligochaeta, Arachnids, Gordiaceae, Nematoda and Bivalves), 20 orders and 75 families comprising 14401 individuals were collected. Taxonomic richness was dominated by insects (84.78\%) divided in eight orders: Coleoptera, Diptera, Ephemeroptera, Hemiptera, Lepidoptera, Odonates, Plecoptera and Trichoptera and 57 families.

A total of 104 different taxa belonging to eight classes (Acheata, Arachnids, Bivalves, Gastropoda, Gordiaceae, Insecta, Malacostraceae, Oligochaeta,), 15 orders and 48 families comprising 3,355 individuals were collected in the locality of Tortiya.

In Lauzoua locality, a total of 142 different taxa belonging to nine classes (Acheata, Arachnids, Bivalves, Gastropoda, Gordiaceae, Insecta, Malacostraceae, Nematoda and Oligochaeta), 19 orders and 65 families comprising 6630 individuals were collected.

In Hiré, 133 taxa belonging to eight classes (Acheata, Arachnids, Bivalves, Gastropoda, Gordiaceae, Insecta, Malacostraceae, Oligochaeta,), 17 orders and 63 families comprising 4,416 individuals were collected.

Taxonomic richness was dominated by insects in all localities (87: Tortiya, 123: Lauzoua, Hiré $=112$ ). The main orders of insects encountered in these localities were: Diptera (Tortiya $=36.13 \%$; Lauzoua $=33.68 \%$ and Hiré $=$ $29.43 \%)$; Odonata $($ Tortiya $=20.74 \%$; Lauzoua $=16.07 \%$ and Hiré $=21.09 \%)$, Coleoptera $($ Tortiya $=15,45 \%$, Lauzoua $=13.31 \%$ and Hiré $=16.02 \%)$. This class was most abundant in Lauzoua $(48.62 \%)$ and Tortiya $(80.59 \%)$. However, macroinvertabrates community was numerically dominated by Gastropods in Hiré (61.50\%). In all the stations, rare taxa were the most abundant except in Lauzoua where they were in very low proportion (6.67\%, N'Téko station). The highest percentage (25.64\%) of very frequent taxa was found in Tortiya (Bou 2 station) and the lowest $(12.50 \%)$ in Hiré (Tributary Gbloh station). Frequent taxa were abundant in Lauzoua precisely in N'Téko station (71.11\%). Their lowest proportion (20.73\%) was found in Hiré (Tchindégri station). 
Table 3. Taxonomic list and occurencies of macroinvertebrates collected; *** (Very frequent taxa), **(Frequent taxa), *(Rare taxa)

Stations codes (D: Dougodou, TD: Tributary Dougodou, NT: N'Teko, G: Gbloh, TG: Tributary Gbloh, Tg:

Tchindégri, B1: Bou1, B2: Bou2)

\begin{tabular}{|c|c|c|c|c|c|c|c|c|c|c|c|}
\hline \multirow[b]{2}{*}{ CLASS } & \multirow[b]{2}{*}{ ORDER } & \multirow[b]{2}{*}{ FAMILIES } & \multirow[b]{2}{*}{ TAXA } & \multicolumn{2}{|c|}{ LAUZOUA } & \multicolumn{3}{|c|}{ HIRE } & \multicolumn{2}{|c|}{ TORTIYA } & \\
\hline & & & & T D & NT & G & TG & $\mathrm{Tg}$ & B1 & $\mathrm{B} 2$ & \\
\hline \multirow[t]{4}{*}{ Acheata } & haryngodelliformes & Erpobdeliidae & Dina sp. & $\mathrm{x}$ & & & & & & & \\
\hline & hynchobdelliformes & Glossiphoniidae & Glossiphonia sp. & $\mathrm{xx}$ & & & $\mathrm{x}$ & & & $\mathrm{x}$ & \\
\hline & & & Haementeria $\mathrm{sp}$. & $\mathrm{x}$ & & & & & & $\mathrm{x}$ & \\
\hline & & & Helobdella sp. & $\mathrm{xxx}$ & $\mathrm{xxx}$ & $\mathrm{x}$ & $\mathrm{xx}$ & $\mathrm{xx}$ & $\mathrm{x}$ & $\mathrm{x}$ & \\
\hline Oligocheata & ind & ind & Oligochètes & $\mathrm{xx}$ & $\mathrm{xx}$ & $\mathrm{x}$ & $\bar{x}$ & $\mathrm{xx}$ & & $\mathrm{xx}$ & \\
\hline \multirow[t]{3}{*}{ Bivalves } & Sphaeriida & Sphaeriidae & Pisidium sp. & $\mathbf{x}$ & & & $\mathbf{x}$ & & & $\mathbf{x}$ & \\
\hline & Unionoida & Corbuliidae & Corbula gibba & $\mathrm{xxx}$ & $\mathrm{xxx}$ & & $\mathrm{xxx}$ & & $\mathrm{x}$ & & \\
\hline & Unionoida & Unionidae & Pseudanodonta sp. & & & & & & & $\mathrm{x}$ & \\
\hline \multirow[t]{7}{*}{ Gastropoda } & Basommatophora & Lymnaeidae & Lymnaea natalensis & $\mathrm{xx}$ & $\mathrm{xxx}$ & $\mathrm{xx}$ & $\mathrm{xxx}$ & $\mathrm{xxx}$ & & $\mathrm{xxx}$ & $\mathrm{xxx}$ \\
\hline & & Physidae & Physa marmorata & $\mathrm{xx}$ & $\mathrm{x}$ & $\mathrm{xx}$ & $\mathrm{xxx}$ & $\mathrm{xxx}$ & & $\mathrm{xx}$ & $\mathrm{xx}$ \\
\hline & & Planorbidae & Biomphalaria pfeifferi & $\mathrm{xxx}$ & $\mathrm{xxx}$ & $\mathrm{xx}$ & $\mathrm{xxx}$ & $\mathrm{xxx}$ & $\mathrm{xx}$ & $\mathrm{xxx}$ & $\mathrm{xx}$ \\
\hline & & & Bulinus forskalii & $\mathrm{x}$ & $\mathrm{xx}$ & $\mathrm{xx}$ & $\mathrm{xx}$ & & $\mathrm{x}$ & $\mathrm{xxx}$ & $\mathrm{xx}$ \\
\hline & & & Bulinus globosus & $\mathrm{xx}$ & $\mathrm{x}$ & & $\mathrm{x}$ & & $\mathrm{x}$ & $\mathrm{xxx}$ & $\mathrm{x}$ \\
\hline & & & Bulinus troncatus & $\mathrm{xx}$ & $\mathrm{xxx}$ & & $\mathrm{xx}$ & & $\mathrm{x}$ & $\mathrm{xxx}$ & $\mathrm{xx}$ \\
\hline & & & Indoplanobis exustus & $\mathrm{xxx}$ & $\mathrm{xxx}$ & $\mathrm{xx}$ & $\mathrm{xx}$ & $\mathrm{xxx}$ & $\mathrm{x}$ & $\mathrm{xxx}$ & $\mathrm{xxx}$ \\
\hline \multirow[t]{6}{*}{ Gastropoda } & Heterostropha & Pyramidellidae & Megastomia conspicua & & & & & & $\mathrm{x}$ & & \\
\hline & Mesogastropoda & Ampullariidae & Lanites varicus & $\mathrm{xx}$ & $\mathrm{xx}$ & $\mathrm{xxx}$ & $\mathrm{X}$ & & $\mathrm{xx}$ & $\mathrm{xx}$ & $\mathrm{xxx}$ \\
\hline & & & Pila africana & $\mathrm{xx}$ & $\mathrm{xxx}$ & $\mathrm{xx}$ & & $\mathrm{xx}$ & $\mathrm{x}$ & & $\mathrm{x}$ \\
\hline & & Bithyniidae & Bythinella sp. & & & & $\mathrm{x}$ & & & & \\
\hline & & & Gabiella africana & $\mathrm{x}$ & $\mathrm{xxx}$ & $\mathrm{xxx}$ & $\mathrm{x}$ & $\mathrm{xx}$ & & & \\
\hline & & Thiaridae & Melanoides tuberculata & $\mathrm{xxx}$ & $\mathrm{xxx}$ & $\mathrm{xxx}$ & $\mathrm{xxx}$ & $\mathrm{xxx}$ & $\mathrm{xxx}$ & $\mathrm{x}$ & $\mathrm{xx}$ \\
\hline Gordiaces & Gordea & Gordiidae & Gordius sp. & $\mathrm{xx}$ & $\mathrm{xx}$ & $\mathrm{xx}$ & $\mathrm{xx}$ & & $\mathrm{x}$ & $\mathrm{x}$ & \\
\hline \multirow[t]{2}{*}{ Nematode } & Hoplonemertea & Tetrastemmatidae & Prostoma sp. & & $\mathrm{x}$ & & & & & & \\
\hline & Mermithida & Mermithidae & Mermithidae & $\mathrm{x}$ & $\mathrm{x}$ & & & & & & \\
\hline Arachnids & Trombidiformes & Hydrachnidae & Hydrachnella sp. & $\mathrm{xxx}$ & $\mathrm{xxx}$ & $\mathrm{xx}$ & $\mathrm{xxx}$ & $\mathrm{xx}$ & $\mathrm{xxx}$ & $\mathrm{xxx}$ & $\mathrm{xxx}$ \\
\hline \multirow[t]{12}{*}{ Insecta } & Coleoptera & Dytiscidae & Dytiscus sp. & & $\mathbf{x}$ & $\mathbf{x x}$ & & & & $\mathbf{x x}$ & $\mathbf{x}$ \\
\hline & & & Hydaticus sp. & $\mathrm{xx}$ & $\mathrm{xx}$ & $\mathrm{xxx}$ & $\mathrm{xx}$ & $\mathrm{X}$ & & $\mathrm{xx}$ & $\mathrm{xxx}$ \\
\hline & & & Hydrocoptus sp. & & $\mathrm{x}$ & & $\mathrm{x}$ & & & $\mathrm{xx}$ & $\mathrm{xx}$ \\
\hline & & & Hydrovatus sp. & $\mathrm{xx}$ & $\mathrm{x}$ & & & & & $\mathrm{xx}$ & \\
\hline & & & Hyphydrus sp. & $\mathrm{x}$ & $\mathrm{x}$ & & $\mathrm{xx}$ & & & & $\mathrm{xx}$ \\
\hline & & & Laccophilus sp. & $\mathrm{xxx}$ & $\mathrm{xxx}$ & & $\mathrm{xx}$ & & $\mathrm{x}$ & $\mathrm{xx}$ & $\mathrm{xxx}$ \\
\hline & & & Neptosternus sp. & $\mathrm{xx}$ & $\mathrm{xx}$ & & & & & & \\
\hline & & Elmidae & Dupophilus sp. & & $\mathrm{x}$ & & & & & & \\
\hline & & & Esolus sp. & $\mathrm{x}$ & $\mathrm{x}$ & & & & $\mathrm{x}$ & & $\mathrm{x}$ \\
\hline & & & Leptelmis sp. & $\mathrm{x}$ & & & $\mathrm{x}$ & & & & \\
\hline & & & Limnius sp. & $\mathrm{xxx}$ & $\mathrm{xxx}$ & & $\mathrm{xx}$ & $\mathrm{xx}$ & $\mathrm{xxx}$ & $\mathrm{x}$ & $\mathrm{xx}$ \\
\hline & & & Macronychus sp. & $\mathrm{x}$ & & $\mathrm{x}$ & & & & & \\
\hline
\end{tabular}


Table 3 continued

\begin{tabular}{|c|c|c|c|c|c|c|c|c|c|c|c|}
\hline \multirow[b]{2}{*}{ CLASS } & \multirow[b]{2}{*}{ ORDER } & \multirow[b]{2}{*}{ FAMILIES } & \multirow[b]{2}{*}{ TAXA } & \multicolumn{3}{|c|}{ LAUZOUA } & \multicolumn{3}{|c|}{ HIRE } & \multicolumn{2}{|c|}{ TORTIYA } \\
\hline & & & & $\mathrm{D}$ & A D & NT & G & $\mathrm{AG}$ & $\mathrm{Tg}$ & B1 & $\mathrm{B} 2$ \\
\hline \multirow[t]{22}{*}{ Insecta } & Coleoptera & Elmidae & Peloriolus sp & & & & & & & $\mathrm{x}$ & \\
\hline & & & Potamodytes sp. & & $\mathrm{xx}$ & & & & $\mathrm{x}$ & $\mathrm{x}$ & \\
\hline & & & Potamophilus sp. & & & & & & $\mathrm{x}$ & & \\
\hline & & & Pseudancryonyx sp. & & & & $\mathrm{x}$ & & & & \\
\hline & & & Riolus sp. & $\mathrm{xx}$ & $\mathrm{xx}$ & & & & & & \\
\hline & & Gyrinidae & Dineutus sp. & & $\mathrm{xx}$ & & $\mathrm{xx}$ & & & & \\
\hline & & & Gyrinus sp. & $\mathrm{x}$ & & & & & & $\mathrm{x}$ & \\
\hline & & & Orectogyrus sp. & $\mathrm{x}$ & $\mathrm{xx}$ & & $\mathrm{x}$ & & $\mathrm{x}$ & & \\
\hline & & Halipidae & Halipus sp. & $\mathrm{x}$ & $\mathrm{x}$ & & $\mathrm{x}$ & & $\mathrm{x}$ & & \\
\hline & & & Peltodytes sp. & $\mathrm{x}$ & $\mathrm{xx}$ & & & & & & \\
\hline & & Hebdidae & Hebdidae & & $\mathrm{x}$ & & & & & & \\
\hline & & & Helodidae & & & & $\mathrm{x}$ & & & & \\
\hline & & Hydraneidae & Hydraena sp. & & $\mathrm{x}$ & & & & $\mathrm{x}$ & $\mathrm{x}$ & $\mathrm{xx}$ \\
\hline & & & Limnebius sp. & $\mathrm{x}$ & & & & & & $\mathrm{x}$ & \\
\hline & & & Mesoceriaton sp. & & & & & & $\mathrm{x}$ & & $\mathrm{x}$ \\
\hline & & Hydrophilidae & Amphiops sp. & $\mathrm{XX}$ & $\mathrm{Xx}$ & $\mathrm{xx}$ & $\mathrm{XXX}$ & & $\mathrm{x}$ & $\mathrm{xxx}$ & $\mathrm{xxx}$ \\
\hline & & & Berosus sp. & & $\mathrm{x}$ & $\mathrm{xx}$ & $\mathrm{x}$ & & & $\mathrm{xx}$ & \\
\hline & & & Enochrus sp. & $\mathrm{x}$ & $\mathrm{x}$ & & $\mathrm{x}$ & & $\mathrm{x}$ & $\mathrm{x}$ & $\mathrm{x}$ \\
\hline & & & Hydrobiinae & & $\mathrm{x}$ & $\mathrm{xx}$ & $\mathrm{xx}$ & $\mathrm{xx}$ & & $\mathrm{xx}$ & $\mathrm{Xx}$ \\
\hline & & & Hydrobius sp. & & $\mathrm{x}$ & & & $\mathrm{x}$ & & $\mathrm{x}$ & $\mathrm{x}$ \\
\hline & & & Hydrochara sp. & & & & $\mathrm{x}$ & $\mathrm{xx}$ & & $\mathrm{xxx}$ & \\
\hline & & & Polyphaga sp. & $\mathrm{xx}$ & $\mathrm{xx}$ & & $\mathrm{x}$ & & & & \\
\hline \multirow[t]{20}{*}{ Insecta } & Coleoptera & Hydroscaphidae & Hydroscapha sp. & & & & & & & & $\mathbf{x}$ \\
\hline & & Noteridae & Noterus sp. & & & & & & & & $\mathrm{x}$ \\
\hline & & Scirtidae & Cyphon sp. & & & & & & & $\mathrm{x}$ & \\
\hline & & & Helodes sp. & & & & & & $\mathrm{xx}$ & $\mathrm{x}$ & \\
\hline & & Athericidae & Atherix sp. & $\mathrm{x}$ & & & & & $\mathrm{x}$ & & \\
\hline & & Blephariceridae & Clogmia sp. & $\mathrm{x}$ & & & & & & & \\
\hline & Diptera & Ceratopogonidae & Atrichopogon sp. & & $\mathrm{x}$ & & $\mathrm{x}$ & & $\mathrm{x}$ & $\mathrm{x}$ & \\
\hline & & & Bezzia sp. & $\mathrm{xx}$ & $\mathrm{xx}$ & & $\mathrm{Xx}$ & & $\mathrm{xxx}$ & $\mathrm{xx}$ & $\mathrm{x}$ \\
\hline & & & Culicoides sp. & & $\mathrm{x}$ & & & & & $\mathrm{x}$ & $\mathrm{x}$ \\
\hline & & Charboridae & Chaoborus sp. & & & & & & $\mathrm{x}$ & $\mathrm{x}$ & \\
\hline & & Chironomidae & Ablabesmyia sp. & $\mathrm{xx}$ & $\mathrm{xxx}$ & & $\mathrm{xx}$ & & $\mathrm{xx}$ & $\mathrm{xxx}$ & $\mathrm{xx}$ \\
\hline & & & Chironomus sp. & $\mathrm{xxx}$ & $\mathrm{xxx}$ & $\mathrm{xx}$ & $\mathrm{xx}$ & $\mathrm{x}$ & $\mathrm{x}$ & $\mathrm{xx}$ & $\mathrm{xx}$ \\
\hline & & & Cladotanytarsus sp. & & & & & $\mathrm{x}$ & & & \\
\hline & & & Clinotanypus sp. & $\mathrm{xx}$ & $\mathrm{xx}$ & & & & $\mathrm{x}$ & & \\
\hline & & & Cricotopus sp. & & & & & & & $\mathrm{x}$ & \\
\hline & & & Cryptochironomus sp. & $\mathrm{xxx}$ & $\mathrm{xx}$ & $\mathrm{xx}$ & $\mathrm{Xx}$ & $\mathrm{xx}$ & $\mathrm{xxx}$ & $\mathrm{xxx}$ & $\mathrm{xxx}$ \\
\hline & & & Nilodorum sp. & $\mathrm{xx}$ & $\mathrm{xx}$ & $\mathrm{xxx}$ & $\mathrm{xx}$ & & $\mathrm{xxx}$ & $\mathrm{xx}$ & $\mathrm{xx}$ \\
\hline & & & Ortocladius sp. & & & & & & & $\mathrm{X}$ & \\
\hline & & & Polypedilium sp. & & $\mathrm{xxx}$ & & $\mathrm{xx}$ & $\mathrm{x}$ & $\mathrm{xx}$ & $\mathrm{xx}$ & $\mathrm{xx}$ \\
\hline & & & Procladius sp. & $\mathrm{x}$ & & & $\mathrm{x}$ & & & $\mathrm{x}$ & \\
\hline
\end{tabular}


Table 3 continued

\begin{tabular}{|c|c|c|c|c|c|c|c|c|c|c|c|}
\hline \multirow[b]{2}{*}{ CLASS } & \multirow[b]{2}{*}{ ORDER } & \multirow[b]{2}{*}{ FAMILIES } & \multirow[b]{2}{*}{ TAXA } & \multicolumn{3}{|c|}{ LAUZOUA } & \multicolumn{3}{|c|}{ HIRE } & \multicolumn{2}{|c|}{ TORTIYA } \\
\hline & & & & $\mathrm{D}$ & A D & NT & $\mathrm{G}$ & $\mathrm{AG}$ & $\operatorname{Tg}$ & $\mathrm{B} 1$ & $\mathrm{~B} 2$ \\
\hline \multirow[t]{21}{*}{ Insecta } & Diptera & Chironomidae & Stictochironomus sp. & $\mathrm{xxx}$ & $\mathrm{xxx}$ & & $\mathrm{xxx}$ & $\mathrm{xx}$ & $\mathrm{xxx}$ & $\mathrm{xxx}$ & $\mathrm{xxx}$ \\
\hline & & & Tanypus sp. & $\mathrm{xx}$ & & & & & $\mathrm{x}$ & & $\mathrm{x}$ \\
\hline & & & Tanytarsus sp. & $\mathrm{x}$ & & & & & & & \\
\hline & & & Xenochironomus sp. & & & & & $\mathrm{x}$ & & & $\mathrm{x}$ \\
\hline & & Culicidae & Aedes sp. & & & & & $\mathrm{x}$ & & $\mathrm{x}$ & \\
\hline & & & Anopheles sp. & $\mathrm{x}$ & $\mathrm{xx}$ & & $\mathrm{x}$ & & & $\mathrm{xx}$ & $\mathrm{x}$ \\
\hline & & & Culex sp. & & $\mathrm{xx}$ & & $\mathrm{xx}$ & $\mathrm{x}$ & & $\mathrm{xx}$ & $\mathrm{xx}$ \\
\hline & & & Culicinae & $\mathrm{x}$ & $\mathrm{xx}$ & & & & & $\mathrm{xx}$ & $\mathrm{xx}$ \\
\hline & & Dixidae & Dixa sp. & & & & & & $\mathrm{x}$ & & $\mathrm{x}$ \\
\hline & & Empididae & Trichoclinocera sp. & $\mathrm{x}$ & & & $\mathrm{x}$ & & & & \\
\hline & & Muscidae & Dolichopodid sp. & & & & & & $\mathrm{x}$ & & \\
\hline & & & Ephydrid sp & $\mathrm{x}$ & & & & & & & \\
\hline & & Psychodidae & Psychodidae & & $\mathrm{x}$ & & $\mathrm{x}$ & & $\mathrm{x}$ & & \\
\hline & & Simulidae & Simulium sp. & $\mathrm{x}$ & $\mathrm{x}$ & & & & $\mathrm{xx}$ & & \\
\hline & & Stratiomyidae & Brachycera sp. & & $\mathrm{x}$ & & $\mathrm{x}$ & $\mathrm{x}$ & & $\mathrm{x}$ & $\mathrm{x}$ \\
\hline & & Syrphidae & Eristales sp. & $\mathrm{x}$ & $\mathrm{x}$ & & & & $\mathrm{x}$ & $\mathrm{x}$ & \\
\hline & & Tabanidae & Tabanus sp. & & $\mathrm{x}$ & & $\mathrm{x}$ & & $\mathrm{x}$ & $\mathrm{x}$ & $\mathrm{x}$ \\
\hline & & Thaumaleidae & Thaumalea sp. & & & & & & $\mathrm{x}$ & $\mathrm{x}$ & $\mathrm{x}$ \\
\hline & & Tipulidae & Limonia tipulipes & $\mathrm{x}$ & & & $\mathrm{x}$ & $\mathrm{x}$ & & & $\mathrm{x}$ \\
\hline & Ephemeroptera & Baetidae & Baetis sp. & $\mathrm{xxx}$ & $\mathrm{xxx}$ & $\mathrm{xxx}$ & $\mathrm{xxx}$ & $\mathrm{xxx}$ & $\mathrm{xxx}$ & $\mathrm{xxx}$ & $\mathrm{xxx}$ \\
\hline & & & Cloeon sp. & $x x$ & $\mathrm{xx}$ & & $\mathrm{xx}$ & & $\mathrm{x}$ & $\mathrm{xx}$ & $\mathrm{xxx}$ \\
\hline
\end{tabular}


Table 3 continued

\begin{tabular}{|c|c|c|c|c|c|c|c|c|c|c|c|}
\hline \multirow[b]{2}{*}{ CLASS } & \multirow[b]{2}{*}{ ORDER } & \multirow[b]{2}{*}{ FAMILIES } & \multirow[b]{2}{*}{ TAXA } & \multicolumn{3}{|c|}{ LAUZOUA } & \multicolumn{3}{|c|}{ HIRE } & \multicolumn{2}{|c|}{ TORTIYA } \\
\hline & & & & $\mathrm{D}$ & A D & NT & $\mathrm{G}$ & $\mathrm{AG}$ & $\mathrm{Tg}$ & $\mathrm{B} 1$ & B2 \\
\hline \multirow[t]{21}{*}{ Insecta } & Ephemeroptera & Baetidae & Pseudocleon sp. & $\mathrm{x}$ & & & & & & & \\
\hline & & Caenidae & Caenis sp. & $\mathrm{xx}$ & $\mathrm{xxx}$ & $\mathrm{XX}$ & $\mathrm{xxx}$ & & $\mathrm{xx}$ & $\mathrm{Xx}$ & $\mathrm{xxx}$ \\
\hline & & Heptageniidae & Afronurus sp. & & & & & & $\mathrm{x}$ & & \\
\hline & & & Electrogena sp. & & $\mathrm{x}$ & & $\mathrm{x}$ & & & & \\
\hline & & Leptophlebiidae & Adenophlebiodes & $\mathrm{x}$ & & & & & $\mathrm{xx}$ & & \\
\hline & & & Choroterpes sp. & $\mathrm{x}$ & & & $\mathrm{x}$ & & & & \\
\hline & Hemiptera & Belostomatidae & Diplonychus sp. & $\mathrm{xxx}$ & $\mathrm{xxx}$ & $\mathrm{xx}$ & $\mathrm{xxx}$ & $\mathrm{x}$ & $\mathrm{x}$ & $\mathrm{xxx}$ & $\mathrm{xxx}$ \\
\hline & & & Limnogeton sp. & $\mathrm{x}$ & $\mathrm{xxx}$ & & & $\mathrm{x}$ & & & \\
\hline & & Gerridae & Eurymetra sp. & $\mathrm{x}$ & $\mathrm{x}$ & & $\mathrm{Xx}$ & $\mathrm{x}$ & $\mathrm{xx}$ & $\mathrm{x}$ & $\mathrm{xx}$ \\
\hline & & & Gerris sp. & & & & & & & & $\mathrm{x}$ \\
\hline & & & Lymnogonus sp. & $\mathrm{x}$ & $\mathrm{xx}$ & & $\mathrm{xx}$ & $\mathrm{x}$ & $x x$ & $\mathrm{xx}$ & $\mathrm{xx}$ \\
\hline & & & Rhagadotarsus sp. & $\mathrm{x}$ & & & & $\mathrm{x}$ & & & \\
\hline & & & Rhagovelia sp. & $\mathrm{xx}$ & $\mathrm{xx}$ & & $\mathrm{x}$ & $\mathrm{x}$ & $\mathrm{x}$ & $\mathrm{x}$ & $\mathrm{x}$ \\
\hline & & Hydrometridae & Hydrometra sp. & & & & & $\mathrm{xx}$ & & $\mathrm{x}$ & \\
\hline & & Mesoveliidae & Mesovelia sp. & $\mathrm{xxx}$ & $\mathrm{xx}$ & $\mathrm{XX}$ & $\mathrm{xx}$ & $\mathrm{x}$ & $\mathrm{xx}$ & $\mathrm{xx}$ & $\mathrm{xx}$ \\
\hline & & Naucoridae & Naucoris sp. & $\mathrm{x}$ & $\mathrm{x}$ & $\mathrm{x}$ & $\mathrm{x}$ & $\mathrm{x}$ & $\mathrm{x}$ & $\mathrm{x}$ & $\mathrm{x}$ \\
\hline & & & Neomacrocoris sp. & & & & $\mathrm{xx}$ & $\mathrm{xx}$ & & $\mathrm{xxx}$ & $\mathrm{xxx}$ \\
\hline & & Nepidae & Laccotrepes sp. & $\mathrm{x}$ & $\mathrm{xx}$ & & $\mathrm{x}$ & $\mathrm{x}$ & & $\mathrm{x}$ & \\
\hline & & Notonectidae & Anisops sp. & $\mathrm{x}$ & $\mathrm{xx}$ & $\mathrm{XX}$ & & $\mathrm{xx}$ & $\mathrm{x}$ & $\mathrm{xx}$ & $\mathrm{xx}$ \\
\hline & & & Enithares sp. & $\mathrm{x}$ & $\mathrm{x}$ & & $\mathrm{x}$ & & & $\mathrm{x}$ & $\mathrm{xx}$ \\
\hline & & & Notonecta sp. & $\mathrm{xx}$ & $\mathrm{x}$ & $\mathrm{xx}$ & $\mathrm{xx}$ & & & $\mathrm{xxx}$ & \\
\hline
\end{tabular}


Table 3 continued

\begin{tabular}{|c|c|c|c|c|c|c|c|c|c|c|c|}
\hline \multirow[b]{2}{*}{ CLASS } & \multirow[b]{2}{*}{ ORDER } & \multirow[b]{2}{*}{ FAMILIES } & \multirow[b]{2}{*}{ TAXA } & \multicolumn{3}{|c|}{ LAUZOUA } & \multicolumn{3}{|c|}{ HIRE } & \multicolumn{2}{|c|}{ TORTIYA } \\
\hline & & & & $\mathrm{D}$ & A D & NT & $\mathrm{G}$ & $\mathrm{AG}$ & $\mathrm{Tg}$ & B1 & $\mathrm{B} 2$ \\
\hline \multirow[t]{21}{*}{ Insecta } & Hemiptera & Pentatomidae & Podisus sp. & $\mathrm{x}$ & & & $\mathrm{x}$ & & & & \\
\hline & & Ranatridae & Ranatra sp. & $\mathrm{x}$ & & & $\mathrm{x}$ & & & & \\
\hline & & Veliidae & Microvelia sp. & $\mathrm{xx}$ & $\mathrm{xx}$ & & $\mathrm{xx}$ & & & $\mathrm{x}$ & $\mathrm{xxx}$ \\
\hline & Lepidoptera & Pyralidae & Elophila sp. & $\mathrm{xx}$ & $\mathrm{xx}$ & $\mathrm{xxx}$ & $\mathrm{x}$ & $\mathrm{xx}$ & & $\mathrm{xx}$ & $\mathrm{xx}$ \\
\hline & Odonata & Calopterygidae & Phaon sp. & & $\mathrm{x}$ & $\mathrm{xx}$ & & & $\mathrm{x}$ & & \\
\hline & & & Sapho bicolor & $\mathrm{x}$ & & & & & & & \\
\hline & & Chlorocyphidae & Chlorocypha sp. & $\mathrm{xx}$ & & & $\mathrm{xx}$ & & $\mathrm{x}$ & $\mathrm{x}$ & \\
\hline & & Coenagrionidae & Ceriagrion sp. & $\mathrm{xx}$ & $\mathrm{x}$ & & $\mathrm{Xx}$ & $\mathrm{xx}$ & $\mathrm{x}$ & $\mathrm{xx}$ & $\mathrm{xxx}$ \\
\hline & & & Coenagrion sp. & $\mathrm{x}$ & & $\mathrm{XX}$ & $\mathrm{XX}$ & & $\mathrm{Xx}$ & & \\
\hline & & & Erythromma sp. & & $\mathrm{x}$ & & & & & & \\
\hline & & & Pseudagrion sp. & $\mathrm{xxx}$ & $\mathrm{xxx}$ & $\mathrm{x}$ & $\mathrm{xxx}$ & XXX & $\mathrm{xxx}$ & $\mathrm{xxx}$ & $\mathrm{xxx}$ \\
\hline & & Corduliidae & Cordulia sp. & $\mathrm{xx}$ & $\mathrm{xx}$ & $\mathrm{xx}$ & $\mathrm{xx}$ & $\mathrm{x}$ & $\mathrm{x}$ & $\mathrm{xx}$ & $\mathrm{xxx}$ \\
\hline & Odonata & Gomphidae & Gomphus sp. & $\mathrm{x}$ & & & & & & $\mathrm{xx}$ & $\mathrm{Xx}$ \\
\hline & & & Ictinogomphus sp. & $\mathrm{xx}$ & $\mathrm{x}$ & & & $\mathrm{x}$ & & & $\mathrm{x}$ \\
\hline & & & Paragomphus sp. & $\mathrm{xx}$ & $\mathrm{xx}$ & & $\mathrm{x}$ & $\mathrm{x}$ & & $\mathrm{x}$ & \\
\hline & & & Phyllogomphus sp. & $\mathrm{x}$ & $\mathrm{XX}$ & & $\mathrm{x}$ & $\mathrm{x}$ & $\mathrm{xxx}$ & & \\
\hline & & Libellulidae & Bradinopyga sp. & $\mathrm{xxx}$ & $\mathrm{xxx}$ & $\mathrm{xx}$ & $\mathrm{xx}$ & $\mathrm{x}$ & & $\mathrm{xxx}$ & $\mathrm{xxx}$ \\
\hline & & & Chalcostephia sp. & & & & & $\mathrm{x}$ & & $\mathrm{x}$ & \\
\hline & & & Diplacodes sp. & $\mathrm{x}$ & & & & & & & \\
\hline & & & Libellula sp. & & & & & & $\mathrm{x}$ & & \\
\hline & & & Oplogastra sp. & $\mathrm{xx}$ & $\mathrm{xxx}$ & $\mathrm{xx}$ & & $\mathrm{x}$ & $\mathrm{x}$ & & $\mathrm{x}$ \\
\hline
\end{tabular}


Table 3 continued

\begin{tabular}{|c|c|c|c|c|c|c|c|c|c|c|c|}
\hline \multirow[b]{2}{*}{ CLASS } & \multirow[b]{2}{*}{ ORDER } & \multirow[b]{2}{*}{ FAMILIES } & \multirow[b]{2}{*}{ TAXA } & \multicolumn{3}{|c|}{ LAUZOUA } & \multicolumn{3}{|c|}{ HIRE } & \multicolumn{2}{|c|}{ TORTIYA } \\
\hline & & & & $\mathrm{D}$ & A D & NT & $\mathrm{G}$ & $\mathrm{AG}$ & $\mathrm{Tg}$ & B1 & B2 \\
\hline \multirow[t]{23}{*}{ Insecta } & Odonata & Libellulidae & Sympertum sp. & & & & & & & & $\mathrm{x}$ \\
\hline & & & Trithemis sp. & & $\mathrm{x}$ & & $\mathrm{x}$ & $\mathrm{x}$ & & & $\mathrm{x}$ \\
\hline & & & Urothemis sp. & & $\mathrm{x}$ & & & $\mathrm{x}$ & & $\mathrm{x}$ & \\
\hline & & & Zygonix sp. & & $\mathrm{x}$ & & $\mathrm{x}$ & & & & \\
\hline & & Macromiidae & Phyllomacronia & $\mathrm{XX}$ & $\mathrm{xxx}$ & $\mathrm{xx}$ & $\mathrm{x}$ & & $\mathrm{x}$ & $\mathrm{xxx}$ & $\mathrm{xx}$ \\
\hline & & & sp. & & & & & & & & \\
\hline & Plecoptera & Perlidae & Neoperla sp. & $\mathrm{x}$ & $\mathrm{x}$ & & $\mathrm{x}$ & & & $\mathrm{x}$ & \\
\hline & Trichoptera & Ecnomidae & Ecnomus sp. & & $\mathrm{xx}$ & & & & & & \\
\hline & & Goeridae & Lithax sp. & & $\mathrm{x}$ & & & & & & \\
\hline & & Glossomatidae & Glossosoma & $\mathrm{x}$ & & & & & & & \\
\hline & & Hydropsychidae & Aethaloptera sp. & & & & & & $\mathrm{x}$ & & \\
\hline & & & Amphipsyche sp. & $\mathrm{x}$ & $\mathrm{Xx}$ & & $\mathrm{x}$ & $\mathrm{x}$ & $\mathrm{xx}$ & & \\
\hline & & & Cheumatopsyche & $\mathrm{XX}$ & $\mathrm{xxx}$ & $\mathrm{xxx}$ & $\mathrm{xx}$ & $\mathrm{xx}$ & $\mathrm{XxX}$ & $\mathrm{x}$ & $\mathrm{x}$ \\
\hline & & & sp. & & & & & & & & \\
\hline & & Hydropsychidae & Chimara sp. & $\mathrm{xx}$ & $\mathrm{x}$ & & $\mathrm{xx}$ & & & & \\
\hline & & & Hydropsyche sp. & $\mathrm{xx}$ & $\mathrm{xx}$ & $\mathrm{xx}$ & $\mathrm{xx}$ & & & $\mathrm{x}$ & $\mathrm{x}$ \\
\hline & & & Polymorphanisus & $\mathrm{xxx}$ & $\mathrm{xxx}$ & $\mathrm{xx}$ & $\mathrm{x}$ & & $\mathrm{x}$ & & \\
\hline & & & Protomacronema & & $\mathrm{x}$ & & & & & & \\
\hline & & Hydroptilidae & Hydroptila sp. & $\mathrm{x}$ & & & & & & & \\
\hline & & & Orthotrichia sp. & $\mathrm{x}$ & & & & & & & \\
\hline & & Leptoceridae & Athripsodes sp. & & $\mathrm{x}$ & & & & & & \\
\hline & & & Ceraclea sp. & $\mathrm{XX}$ & $\mathrm{xx}$ & & $\mathrm{x}$ & $\mathrm{x}$ & & & \\
\hline & & & Leptocerus sp. & & & & & & $\mathrm{x}$ & & \\
\hline
\end{tabular}


Table 3 continued

\begin{tabular}{|c|c|c|c|c|c|c|c|c|c|c|c|}
\hline \multirow[b]{2}{*}{ CLASS } & \multirow[b]{2}{*}{ ORDER } & \multirow[b]{2}{*}{ FAMILIES } & \multirow[b]{2}{*}{ TAXA } & \multicolumn{3}{|c|}{ LAUZOUA } & \multicolumn{3}{|c|}{ HIRE } & \multicolumn{2}{|c|}{ TORTIYA } \\
\hline & & & & $\mathrm{D}$ & A D & NT & G & $\mathrm{AG}$ & $\mathrm{Tg}$ & B1 & B2 \\
\hline \multirow[t]{5}{*}{ Insecta } & Trichoptera & Leptoceridae & Parasetodes sp. & $\mathrm{x}$ & & $\mathrm{xx}$ & $\mathrm{x}$ & & $\mathrm{xx}$ & & \\
\hline & & & Setodes sp. & $\mathrm{xxx}$ & $\mathrm{xx}$ & & & & & & \\
\hline & & & Trianodes sp. & & $\mathrm{x}$ & & & & $\mathrm{x}$ & $\mathrm{x}$ & \\
\hline & & & Trichosetodes & & & & & & $\mathrm{x}$ & & \\
\hline & & Polycentropodidae & Polycentropus & & $\mathrm{x}$ & & & & & & \\
\hline \multirow[t]{11}{*}{ Malacostracea } & Decapoda & Atyidae & $\begin{array}{l}\text { Caridina } \\
\text { africana }\end{array}$ & $\mathrm{xxx}$ & $\mathrm{xxx}$ & $\mathrm{xxx}$ & $\mathrm{xxx}$ & $\mathrm{x}$ & $\mathrm{xxx}$ & $\mathrm{xx}$ & $\mathrm{x}$ \\
\hline & & & Caridinopsis & & & & & & $\mathrm{x}$ & & \\
\hline & & Desmocarididae & $\begin{array}{c}\text { Desmocaris } \\
\text { trispinosa }\end{array}$ & $\mathrm{x}$ & & & $\mathrm{x}$ & & & & \\
\hline & & Palaemonidae & Macrobrachium & & & $\mathrm{xx}$ & & & & & \\
\hline & & & Thysi & & & & & & & & \\
\hline & & & Macrobrachium & $\mathrm{x}$ & & $\mathrm{xx}$ & & & $\mathrm{x}$ & & \\
\hline & & & Vollenhovenii & & & & & & & & \\
\hline & & Potamonautidae & Liberonautes & & $\mathrm{x}$ & $\mathrm{xx}$ & & & $\mathrm{xxx}$ & & \\
\hline & & & chaperi & & & & & & & & \\
\hline & & & Potamon sp. & & $\mathrm{xx}$ & & $\mathrm{xx}$ & & $\mathrm{x}$ & & \\
\hline & & & Potamonautes & & & & & & $\mathrm{x}$ & & \\
\hline \multicolumn{2}{|c|}{ Taxonomic richness } & & & 107 & 111 & 46 & 91 & 55 & 81 & 86 & 76 \\
\hline
\end{tabular}

\subsection{Macroinvertebrate Structure}

Figure 2 shows variations of diversities indices between stations. The rarefied richness in Lauzoua was ranged between 2.96 (N'Téko station) and 8.34 (Dougodou station). This index was significantly higher in Dougodou station (Mann- Whitney test $\mathrm{p}<0.05$ ). In Hiré, rarified richness was between 2.34 (Gbloh station) and 7.23 (Tchindégri station). This index was significantly lower at Gbloh station (Mann- Whitney test $\mathrm{p}<0$. 05). Concerning Tortiya, this index oscillated between 3.37 and 8.75 (Bou 2 station). 

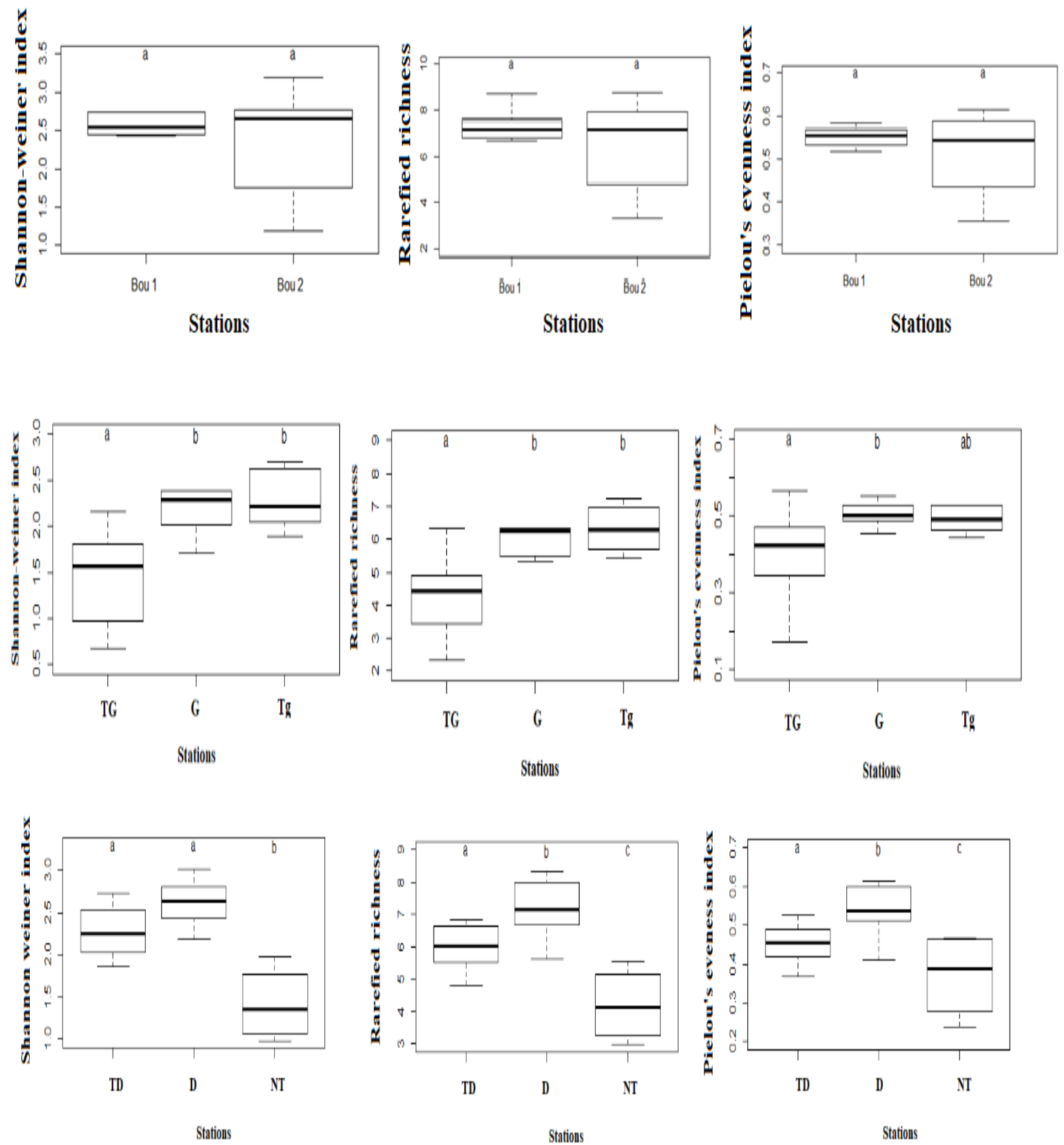

Figure 2. Boxplots showing differences in diversity indices (rarefied richness, Shannon Weiner, Pielou's evenness index) between sampling stations in three mining areas in Côte d'Ivoire. Different letters (a,b,c) on Boxplots denote significant differences between them (Mann- Whitney test, $\mathrm{P}<0.05)$. B1- B2= TORTIYA,

TD-NT= LAUZOUA, TG-Tg= HIRE

Shannon-Weiner index observed in Lauzoua, varied between 0.98 (N'Téko station) and 3.02 (Dougodou station). This index was significantly higher in Dougodou station (Mann- Whitney test $\mathrm{p}<0.05$ ). In Hiré, it was located between 0.68 (Tributary Gbloh station) and 2.69 (Tchindégri station). Shannon-Weiner index was significantly lower in Tributary Gbloh station (Mann- Whitney test $\mathrm{p}<0$. 05). Regarding Tortiya, this index was ranged between 1.19 (Bou 2 station) and 3.3 (Bou 1 station).

The Pielou's Evenness Index in Lauzoua varied from 0.24 (N'Téko station) to 0.61 (Dougodou station). This index was significantly lower in N'Téko station (Mann- Whitney test $p<0$. 05). In Hiré, this index oscillated between 0.17 and 0.56 (Tributary Gbloh station) and was significantly lower in Tributary Gbloh station (MannWhitney test $\mathrm{p}<0.05$ ). In Tortiya, this parameter varied from 0.36 to 0.62 (Bou 2 station). 
In Lauzoua, EPT percentage varied between $4.50 \%$ (N'Téko station) and $18.92 \%$ (Tributary Dougodou station). In the locality of Tortiya, the value of ETP index was $13 \%$ at the two stations (Bou 1 and Bou 2). Relative abundance of EPT was less than $6 \%$ in Hiré at all stations.

\subsection{Abiotic and Taxonomic Differentiations of Sampling Stations}

Principal component analysis (PCA) has established the abiotic typology of the stations studied (Figure 3). The first two axes expressed $52.4 \%$ of the total variance, $39.5 \%$ for axis 1 and $12.9 \%$ for axis 2 (Figure $3 \mathrm{~A}$ ). The correlation circle (Figure 3B) revealed that all of the physico-chemical parameters were negatively correlated to axis 1 excepted the temperature which was positively correlated to axis 2. The factor map (Figure 3C) distinguishes four groups of stations (Figure 3D).
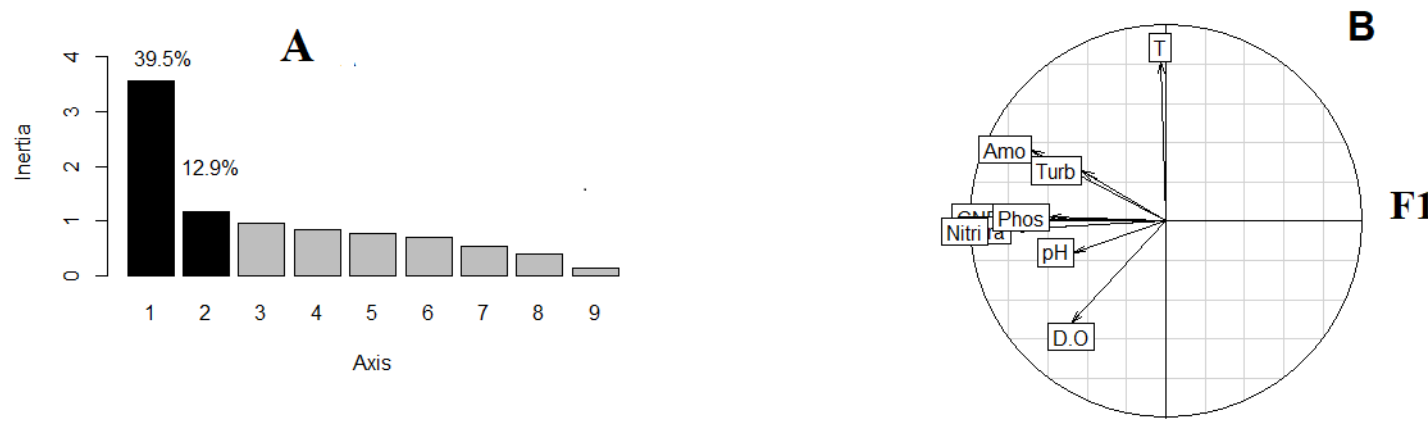

F1

F2

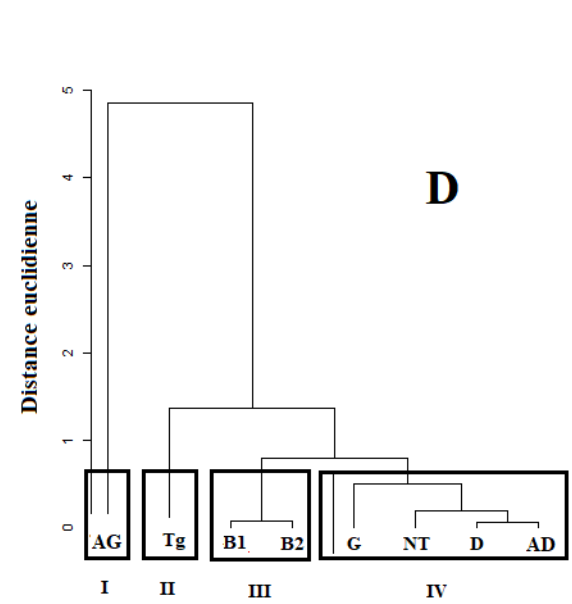

F2

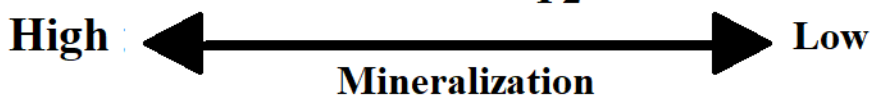

Low

Figure 3. Ordination of physicochemical parameters of the stations studied from a Principal Component Analysis:

$\mathrm{TG}$ to $\mathrm{Tg}=$ Stations codes $; \mathrm{A}=$ histogram of eigen values; $\mathrm{B}=$ circle of correlation; $\mathrm{C}=$ factoriel map; $\mathrm{D}=$

Hierarchical classification, from a Principal Component Analysis : I, II, III and IV = clusters. Amo = Ammonium; DO = Dissolved oxygen; Turb= Turbidity, Phos= Phosphate, CND= Conductivity.

Shannon Weiner index in cluster I ranged from 0.67 to 2.15. Concerning cluster II, this index was between 1.89 and 2.68. Shannon Weiner index of Group III was ranged between 2.48 and 2.74. The Shannon Weiner index of group IV ranged from 1.16 to 3.02 .

Rarefied richness of group I ranged from 2.34 to 6.33. Rarefied richness index of Group II was between 5.41 and 7.23. Rarefied richness of Group III was ranged between 6.71 and 8.7. Rarefied richness of group IV ranged 
from 3.02 to 8.34. Rarefied richness and Shannon Weiner's index were significantly lower in cluster I (Mann Whitney test, $\mathrm{P}<0.05$ ) (Figure 4).
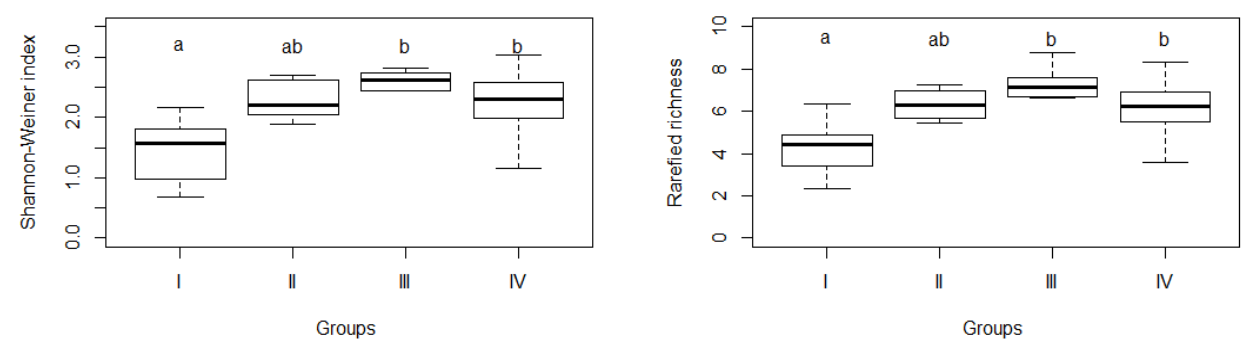

Figure 4. Rarefied richness and Shannon Weiner index of the four clusters obtained after the Hierarchical Classification Analysis. Different letters (a, b) on Boxplots denote significant differences between them (MannWhitney test, $\mathrm{P}<0.05)$.

Cluster I consisted of samples from Tributary Gbloh station. It was characterized by higher values (MannWhitney test $\mathrm{p}<0.05)$ of conductivity $(1214.5-1753)$, ammonium $(2-4.8 \mathrm{mg} / \mathrm{L})$, phosphate, nitrites and nitrates than those obtained in other clusters. Clusters I and II (samples of Tchindégri station) were characterized by higher values (Mann- Whitney test $\mathrm{p}<0.05$ ) of phosphate, nitritres and nitrates comparatively of clusters III and IV. Concerning cluster III, it consisted of samples from stations Bou 1 and Bou 2. This group was characterized by higher values of temperature $\left(24-33.1^{\circ} \mathrm{C}\right)$ compared to the clusters II and IV (Figure 5). Stations of Group IV were characterized by low mineralization.
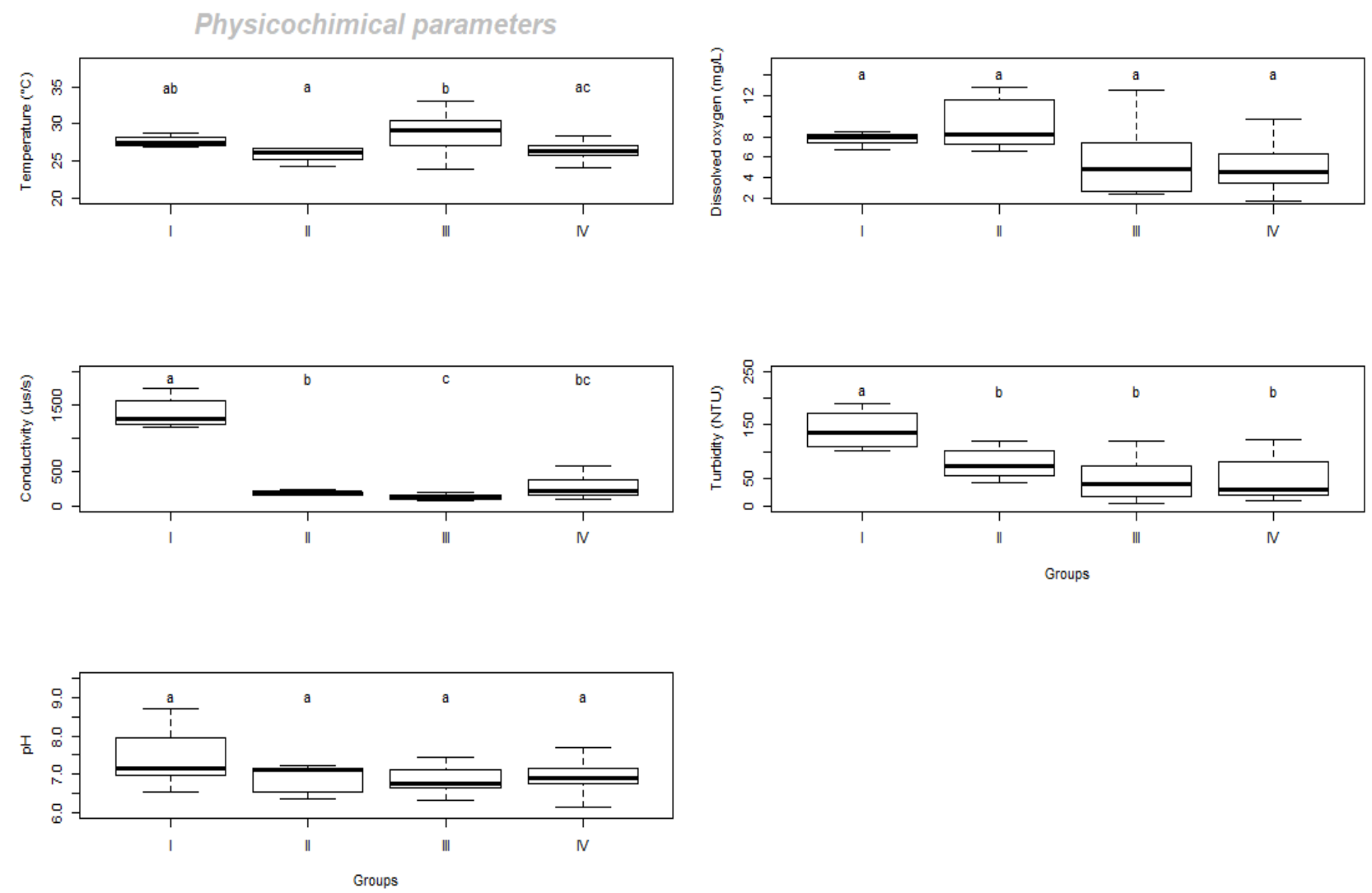

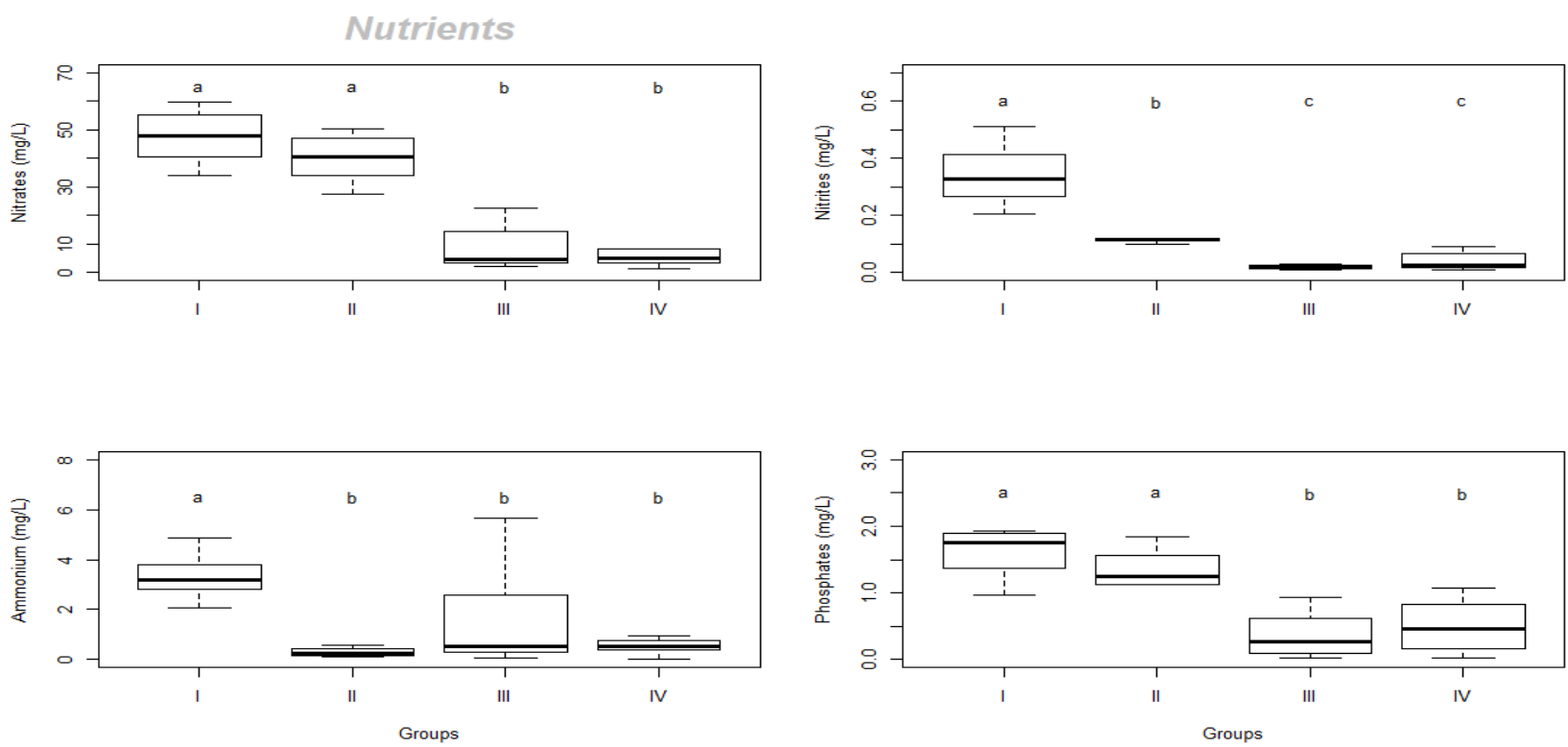

Figure 5. Boxplots showing differences in physicochemical parameters and nutrients concentrations between clusters (I-IV). Different letters (a, b, c) on Boxplots denote significant differences between them (MannWhitney test, $\mathrm{P}<0.05)$.

Indval method revealed that eight taxa (Limnius sp., Liberonautes chaperi, Gordius sp., Phyllogomphus sp., Orectogyrus sp., Bezzia sp., Adenophlebiodes, Parasedodes sp.) were specific to the station Tchindégri (Hiré) and six taxa (Naucoris sp., Amphiops sp., Hydrobius sp., Pseudobagous longulus, Culicinae and Gomphus sp.) were associated at stations in the locality of Tortiya.

\section{Discussion}

During this study, the highest values of temperature were recorded in the stations of Tortiya locality. This could be due to the absence of canopy at these sampling stations. Indeed, any surface of water not covered is subject to a very important sunning thus favoring the increase of the temperature of the water.

The higher values of conductivity (1162 to $1753 \mu \mathrm{S} / \mathrm{cm}$ ) registered in Tributary Gbloh station (Hiré) could be linked to the fact that this station was in an area subject to a permanent supply of effluent favoring the dissolution of the metals present in the rocks and sediments. These results corroborate those of Yapi et al. (2014) in this same locality.

Turbidity was higher in Tributay Gbloh station (102.3 to 189.3 NTU: Hiré). This situation could be explained by the location of this stream in downstream which receive particles from upstream areas where mining activities are practiced. These results corroborate those of Bamba et al. (2013) on the impact of mining on rivers which can destabilize the banks and lead to a massive sediment supply which can locally disturb the balance of the rivers and increase the turbidity of the water.

The abiotic typology of the stations by the PCA revealed that the Triblutary Gbloh station (cluster I) is distinguished from the other stations by high values of the mineralization parameters (conductivity, ammonium, nitrate, nitrite, phosphate). This high mineralization observed in this station could be explained by the drainage of agricultural products in this watercourse. Indeed, according to Brugneaux et al. (2004) and Troeh et al. (2004), by the action of rain that drains cultivated land, surface waters receive increased nutrient inputs.

The Indval method revealed that among the indicator taxa of Tchnidégri station, there were two polluo-sensitive organisms: Adenophlebiodes (Ephemeroptera, tolerance level $=2)$, Parasetodes sp. $($ Trichoptera, tolerance level $=$ 4) recognized as good bio-indicators of watercourses because of their sensitivity to oxygen depletion (Hynes, 1957). We can therefore deduce that the waters of this station have a acceptable ecological quality.

A total of 184 aquatic macroinvertebrate taxa were collected and insects accounted for $84 \%$ of the taxa collected. Insects were the most abundant group among the macroinvertebrates collected in this study. Insects abundance 
could be explained by their omnipresence, which is due to their capacity for resilience. In several studies insects were most abundant (Diomandé et al., 2009; Akindele \& Liadi, 2014).

In Hiré, the lowest rarefied richness (2.34) was obtained at Gbloh station. This station had the lowest values of Shannon Weiner index (0.68) and Pielou's eveness (0.17) index. These results obtained could indicate that this station was the most impacted by gold mining, which would have an impact on aquatic macroinvertebrate assemblages. According to Rosenberg \& Resh (1993), human perturbations change community structure in watercourses because species are adapted to certain environmental conditions. The low diversity in this station may reflect the response of benthic macroinvertebrates to the toxicity in this station. This could be attributed to the loss of habitat diversity due to the reduction of ecological niches. The highest values of the Shannon Weiner index were obtained in Lauzoua and Tortiya respectively at Dougodou (3.02) and Bou 1 (3.2) stations. These results show that the aquatic macroinvertebrates of these stations were most diversified which could reflect good water quality at these stations.

EPT taxa are sensitive macroinvertebrates. They are met usually in water of good quality. This group were present at all stations with different proportions. These organisms were mainly composed of Baetidae (Baetis sp, Ephemeroptera) and Hydropsychidae (Hydropsyche sp., Trichoptera). These organisms are sensitive to metal pollution (Malmquist \& Hoffsten, 1999) but can recolonized rapidly disturbed stations (Kiffney \& Clements, 1994). The Tributary Gbloh station was the most affected by gold mining in Hiré with an EPT proportion of $4.91 \%$. In Lauzoua, N'Téko station recorded the lowest proportion $(4.50 \%)$ of EPT. However, there was no mining activity at this station. The low proportion of EPT at this station may be due to other human activities. In Tortiya locality, EPT proportions were relatively higher: 13.62\% in Bou 1 and 12.30\% in Bou 2. Diamond mining has little impact on station water quality. These proportions obtained could be explained by the fact that no metal is used in diamond mining. One of the limitations of this study is the number of sampling points chosen which should have been several on each station instead of choosing only one sampling point. However, the results show plausible consequences of mining on the quality of rivers, therefore alert the authorities to provide guidance on the consumption of drinking water by populations near these sites.

\section{Conclusion}

This study allowed to collect macroinvertebrates from three mining areas. Gold and manganiferous mining had an impact on macroinvertebrate communities. However, this impact was more significant in the streams near mining operation. Comparing to the latter two mining sites, diamond mining causes least disturbances to macroinvertebrate communities. Therefore, gold and manganiferous mining have most impact on the ecological quality of the studied rivers. Based on findings, actions must be conducted in these mining areas in order to stop the impact of the tailings on the pollution of waters surrounding residential areas.

\section{Acknowledgement}

This paper was produced as part of a project entitled "Ecological quality of the rivers of the mining areas of TORTIYA (Diamond area), LAUZOUA (Manganese area) and HIRE (Gold area) (Ivory Coast)", financed by the United Nations Educational, Scientific and Cultural Organization / SIDA (UNESCO-SIDA).

\section{References}

Akindeke, O. E., \& Liadi, A. A. (2014). Diversity and response of benthic macroinvertebrates to natural and induced environmental stresses in aiba stream, iwo, south western Nigeria. West African Journal of Applied Ecology, 22(1), 101-111.

Bamba, O., Pelede, S., Sako, A., Kagambega, N., \& Miningou, M. Y. (2013). Impact de l'artisanat minier sur les sols d'un environnement agricole aménagé au Burkina Faso. Journal des Sciences, 13(1), 1-11.

Battaglia, M., Hose, G., Turak, E., \& Warden, B. (2005). Depauperate macroinvertebrates in a mine affected stream: clean water may be the key to recovery. Environmental Pollution, 138(1), 132-141.

Bode, R. W., Novak, M. A., \& Abele, L. E. (1996). Quality Assurance Work Plan for Biological Stream Monitoring in New York State, Albany (New York), NYS Department of Environmental Conservation, p. 89.

Bode, R. W., Novak, M. A., Abele, L. E., Heitzmzn, D. L., \& Smith, A. J. (2002). Quality Assurance Work Plan for Biological Stream Monitoring in New York State, Albany (New York), Stream Biomonitoring Unit Bureau of Water Assessment and Management Division of Water, NYS Department of Environmental Conservation, p. 41. Retrieved from http://www.dec.state.ny.us/website/dow/bwam/sbuqa02.pdf 
Brigde, G. (2004). Contested terrain: Miningand the environment. Annual Review of Environment and Resources, 29, 205-259.

Brugneaux, S., Pierret, L., \& Mazataud, V. (2004). Les agressions d'origine anthropique sur le milieu marin côtier et leurs effets sur les écosystèmes coralliens et associés de la Martinique. Les Cahiers de l'Observatoire, $\mathrm{N}^{\circ} 1$, Fort de France: Observatoire du milieu marin martiniquais, p. 96.

Clarke, R. T., Furse, M. T., Gunn, R. J., Winder, J. M., \& Wright, J. F. (2002). Sampling variation in macroinvertebrate data and implications for river quality indices. Freshwater Biology, 38, 327-342.

Coulibaly, A. S., Mondé, S., Wognin, V. A., \& Aka, K. (2009). Analyse des éléments traces métalliques (ETM) dans les baies estuariennes d'Abidjan en Côte d'ivoire. Afrique Sciences, 5(3), 77-96.

Dajoz, R. (2000). Précis d'Ecologie. Edition Dunod, Paris, pp. 373-390.

Day, J. A., Stewart, B. A., \& de Moor, I. J (2001). Guide to the Freshwater Invertebrates of Southern Africa. Crustacea III: Bathynellacea, Amphipoda, Isopoda, Spelaeogriphacea, Tanaidacea, Decapoda. Rapport $N$ TT 141/01 Water Research Commission, South Africa, 4, 141.

Day, J. A., Stewart, B. A., \& de Moor, I. J. (2003). Guide to the Freshwater Invertebrates of Southern Africa. Diptera. Rapport N TT 201/02 Water Research Commission, South Africa, 9, 200.

De Moor, I. J., Day, J. A., \& Moor, F. C. (2003a). Guide to the Freshwater Invertebrates of Southern Africa. Insecta I: Ephemeroptera, Odonata and Plecoptera. Rapport $N^{\circ}$ TT 207/03 Water Research Commission, South Africa, 7, 288.

De Moor, I. J., Day, J. A., \& Moor, F. C. (2003b). Guide to the Freshwater Invertebrates of Southern Africa. Insecta II: Hemiptera, Megaloptera, Neuroptera, Trichoptera and Lepidoptera. Rapport $\mathrm{N}^{\circ}$ TT 214/03 Water Research Commission, South Africa, 8, 209.

Déjoux, C., Elouard, J. M., Forge, P., \& Malsin, J. (1981). Catalogue iconographique des insectes aquatiques de Côte d'Ivoire. Rapport ORSTOM. Bouaké, Côte d'Ivoire, p. 172.

Diomandé, D., Bony, K. Y., Edia, O. E., Konan, F. K., \& Gourène, G. (2009). Diversité des macroinvertébrés benthiques de la rivière Agnéby (Côte d'Ivoire ; Afrique de l'Ouest). European Journal of Scientific Research, 35(3), 368-377.

Dold, B. (2014). Evolution of Acid Mine Drainage Formation in Sulphidic Mine Tailings, 4, 621-641.

Dufrêne, M., \& Legendre, P. (1997). Species assemblages and indicator species: the need for a flexible asymmetrical approach. Ecological Monograph, 67(3), 345-366.

Edia, O. E., Emmanuel, C., Konan, K. M., \& Gattolliat, J. L. (2016). Diversity, distribution and habitat requirements of aquatic insect communities in tropical mountain streams (South-eastern Guinea, West Africa). International Journal of Limnology, 52, 285-300.

Fair, L. (2013). Rapport de l'Administrateur indépendant de l'Initiative pour la Transparence des Industries Extractives (ITIE) pour les revenus de l'année 2011, Côte d'Ivoire, p. 24.

Gray, D. P., \& Harding, J. S. (2012). Acid Mine Drainage Index (AMDI): a benthic invertebrate biotic index for assessing coal mining impacts in New Zealand streams. N Z J Marine Freshwater Research, 46(3), 335-352.

Heck, K. L., Vanbelle, G., \& Simberloff, D. (1975). Explicit calculation of rarefaction diversity measurement and determination of sufficient sample size. Ecology, 56, 1459-1461.

Hilsenhoff, W. L. (1988). Rapid field assessment of organic pollution with a family-level biotic index. Journal of North American Benthological Society, 7(1), 65-68.

Hyslop E. J. (1980). Stomach contents analysis, a review of methods and their application. Journal of Fish Biology, 17, 411-429.

Jennings, S. R., Neuman, D. R., \& Blicker, P. S. (2008). Acid Mine Drainage and Effects on Fish Health and Ecology: A Review. Reclamation Research Group Publication, 29.

Keumean, K. N., Bamba, S. B., Soro, G., Soro, N., Métongo, B. S., \& Biémi, J. (2013). L'étude de l'impact d'un site minier abandonné dans la haute Moulouya sur la qualité de l'Oued Moulouya, Maroc. Afrique Sciences, $7(3), 34-48$. 
Kiffney, P. M., \& Clements, W. H. (1994). Effects of heavy metals on a macroinvertebrate assemblage from a Rocky Mountain stream in experimental microcosms. Journal of the North American Benthological Society, 13, 511-523.

Koffi, Y. B., Kouassi, E. A., Kouassi, M. A., \& Biemi, J. (2014). Mines, oil and gas in Ivory Coast and the problem of Water resources pollution and flooding. International Journal of Tropical Geology, Geography and Ecology., 38(1), 119-136.

Lauzanne, L. (1976). Régimes alimentaires et relations trophiques des poisons du lac Tchad. Cahier de l"ORSTOM, série Hydrobiologie, 10, 267-310.

Malmquist, B., \& Hoffsten, P. (1999). Influence of drainage from old mine deposits on benthic macroinvertebrate communities in central Swedish streams. Water Research, 3, 2415-2423.

McTammany, M. (2007). The impact of episodic coal mine drainage pollution on benthic macroinvertebrates in streams in the Anthracite region of Pennsylvania. Environmental Pollution, 149, 216-226.

Monod, T. (1966). Crevettes et crabes des côtes occidentales d'Afrique. In Gordon, I.(Ed.), Réunion de spécialistes C. S. A. sur les Crustacés (Vol. 77, 103-234). Mémoires de 1'Institut Fondamental d'Afrique Noire, Zanzibar.

Moretti, M. S., \& Callisto, M. (2005). Biomonitoring of benthic macroinvertebrates in the middle Doce River watershed. Acta Limnologica. Brasiliensia, 17(3), 267-281.

Oksanen, J., Blanchet, F. G., Kindt, R., Legendre, P., Minchin, P. R., O’Hara, R. B., .. Wagner, H. (2013). Vegan: Community Ecology Package. R package version 2.0.

Paquet, J. (2012). Analyse des impacts de la mondialisation sur l'économie au Québec. Rapport, 15.

Rosenberg, D. M., \& Resh, V. H. (1993). Freshwater Biomonitoring and Benthic Macroinvertebrates. Springer Publisher, p. 488.

Sanogo, S., Tinkoudgou, K. T. A., \& Cecchi, P. (2014). Inventaire et distribution spatio-temporelle des macroinvertébrés bioindicateurs de trois plans d'eau du bassin de la Volta au Burkina Faso. The International Journal of Biological and Chemical Sciences, 8(3), 1005-1029.

Sodemi. (2012). Rapport d'activité annuel, p. 60.

Stals, R., \& De Moor, I. J. (2007). Guides to the Freshwater Invertebrates of Southern Africa. Coleoptera. Rapport N TT 320/07 Water Research Commission, South Africa, 10, 263.

Tachet, H., Richoux, P., Bournaud, M., \& Usseglio-Polatera, P. (2010). Invertébrés d'eau douce: systématique, biologie, écologie, nouvelle édition revue et augmentée. Paris, CNRS Éditions, p. 607.

Troeh, F. R., Hobbs, J. A., \& Donahue, R. L. (2004). Soil and water conservation: for productivity and environmental protection (4th ed.). Prentice Hall, Upper Saddle River, New Jersey, USA, p. 610.

World Bank. (2019). World Bank Commodities Price Forecast (nominal US dollars). Retrieved from http://pubdocs.worldbank.org/en/598821555973008624/CMO-April-2019-Forecasts.pdf

Yapi, Y. H. A., Dongui, B. K., Trokourey, A., Barima, Y. S. S., Essis, Y., \& Atheba, P. (2014). Evaluation de la pollution métallique des eaux souterraines et de surface dans un environnement minier aurifère à Hiré (Côte d'Ivoire). International journal of biological and chemical sciences, 8(3), 1281-128.

\section{Copyrights}

Copyright for this article is retained by the author(s), with first publication rights granted to the journal.

This is an open-access article distributed under the terms and conditions of the Creative Commons Attribution license (http://creativecommons.org/licenses/by/4.0/). 